\title{
THE RISE OF KINK-UNSTABLE MAGNETIC FLUX TUBES IN THE SOLAR CONVECTION ZONE
}

\author{
Y. FAN \\ High Altitude Observatory, National Center for Atmospheric Research, P.O. Box 3000, Boulder, CO 80307-3000 \\ E. G. ZWEIBEL \\ JILA, University of Colorado at Boulder, Campus Box 440, Boulder, CO 80309-0440 \\ AND \\ M. G. Linton AND G. H. FisheR \\ Space Sciences Laboratory, University of California, Berkeley, Berkeley, CA 94720 \\ Received 1998 June 4; accepted 1998 July 21; published 1998 August 24
}

\begin{abstract}
We report preliminary results of a three-dimensional simulation of the buoyant rise of a strongly twisted, kinkunstable magnetic flux tube through a gravitationally stratified layer representing the solar convection zone. The numerical calculations employ the well-known anelastic approximation, which is suitable for studying slow, subsonic dynamical processes in the pressure-dominated, high- $\beta$ plasma of the solar interior. This Letter investigates the case in which the initial twist of the buoyant flux tube is sufficiently high that the $e$-folding growth times of the unstable kink modes are short in comparison to the rise time of the flux tube. Our simulation shows that the flux tube becomes kinked and that the top portion of the flux tube evolves into a buckled shape with the tube axis being deflected by more than $90^{\circ}$ from its original orientation. We suggest that the emergence of this buckled flux tube can give rise to a compact magnetic bipole with polarity order inverted from Hale's polarity law, similar to the configuration often seen in $\delta$ spots.
\end{abstract}

Subject headings: MHD - Sun: interior — Sun: magnetic fields

\section{INTRODUCTION}

There is mounting evidence from both observations (e.g., Tanaka 1991; Lites et al. 1995; Leka et al. 1996) and theoretical considerations (e.g., McClymont \& Fisher 1989; Low 1996; Moreno-Insertis \& Emonet 1996; Fan, Zweibel, \& Lantz 1998; Matsumoto et al. 1998) that suggests the direct emergence of current-carrying (or twisted) magnetic flux tubes from the solar interior. Through careful analysis of magnetic and velocity data of two flare-active $\delta$ spot groups, Tanaka (1991) inferred a model of the emergence of a twisted magnetic knot to explain the evolution of the $\delta$ groups. The possibility of kinked flux tubes as the origin of $\delta$ spots has recently been investigated by Linton, Longcope, \& Fisher (1996) and Linton et al. (1998a, 1998b), who performed linear and nonlinear calculations of the kink instability of a twisted magnetic flux tube in a uniform fluid without the effects of gravity and stratification. In an effort to study the dynamic evolution of emerging magnetic flux tubes in the solar convection zone, we have developed a three-dimensional anelastic MHD code. This code enables us to model more realistically the nonlinear evolution of the kink instability of a twisted magnetic flux tube as it rises buoyantly through a gravitationally stratified layer. In this Letter, we describe the evolution of a buoyant flux tube that has an initial twist that is significantly above the critical limit for the onset of the kink instability.

\section{THE PHYSICAL PROBLEM AND NUMERICAL METHOD}

The linear kink instability of a twisted magnetic flux tube in the ideal MHD limit has been studied in numerous previous investigations (see Linton et al. 1996 and references therein). For the calculations in this Letter, the initial twisted flux tube has the form $\boldsymbol{B}=B_{\theta}(r) \hat{\theta}+B_{x}(r) \hat{\boldsymbol{x}}$ and

$$
\left\{\begin{array}{l}
B_{x}(r)=B_{x 0} \exp \left(-r^{2} / a^{2}\right) \\
B_{\theta}(r)=\alpha r B_{x}(r)
\end{array}\right.
$$

where $\hat{\boldsymbol{x}}$ denotes the tube axial direction, $\hat{\theta}$ is the azimuthal direction in the tube cross section, and $r$ is the radial distance to the central axis. The flux tube is uniformly twisted, with the constant $\alpha$ denoting the amount of field line rotation about the central axis per unit length of the tube. Such a twisted flux tube is unstable to helical motion of the form $\boldsymbol{v}(r) \exp [i(k x+\theta)]$, if $\alpha>\alpha_{c}=1 / a$. Figure 1 shows the growth rates of the unstable kink modes for $\alpha=4 \alpha_{c}$, computed by solving the Newcomb equation (see, e.g., Freidberg 1982; Linton et al. 1996). It can be seen that there is a range of axial wavenumbers $k$ on either side of $k=-\alpha$ that are unstable. Our numerical simulations have finite viscosity and resistivity, so here we investigate only the long-wavelength range of unstable modes (with $|k|<\alpha$ ), which are less affected by the presence of dissipation. The maximum growth rate of the kink modes yields an $e$-folding growth time of $\tau_{\min } \approx 0.6\left(a / v_{\mathrm{A}_{0}}\right)$, where $v_{\mathrm{A}_{0}}$ is the initial Alfvén speed at the tube center. The growth rates of the kink modes depend upon the value of $\alpha$ (see Linton et al. 1996, in which $q$ is used in place of $\alpha$ ). For example, if $\alpha$ is reduced to $1.2 \alpha_{c}$, then $\tau_{\min }$ is increased to $14\left(a / v_{\mathrm{A}_{0}}\right)$. In comparison, the typical rise time of a magnetic flux tube through the convection zone is estimated to be $\tau_{\text {rise }} \sim H_{p} / v_{\text {rise }}$, where $H_{p}$ is the pressure scale height and the rise velocity $v_{\text {rise }}$ is $\sim\left(a / H_{p}\right)^{1 / 2} v_{\mathrm{A}_{0}}$ (Parker 1975). If the radius of the flux tube $a$ is $\sim 0.1 H_{p}$, then $\tau_{\text {rise }} \sim 30\left(a / v_{\mathrm{A}_{0}}\right)$. This comparison shows that if $\alpha$ is sufficiently large (say above $1.2 \alpha_{c}$ ), we can expect to see significant distortion of the flux tube by the kink modes during the rise of the tube through the convection zone. In this Letter, we present the simulation results for the case in which the initial flux tube has such a high degree of twist ( $\alpha=4 \alpha_{c}$ ) that the $e$-folding time of the fastest growing 


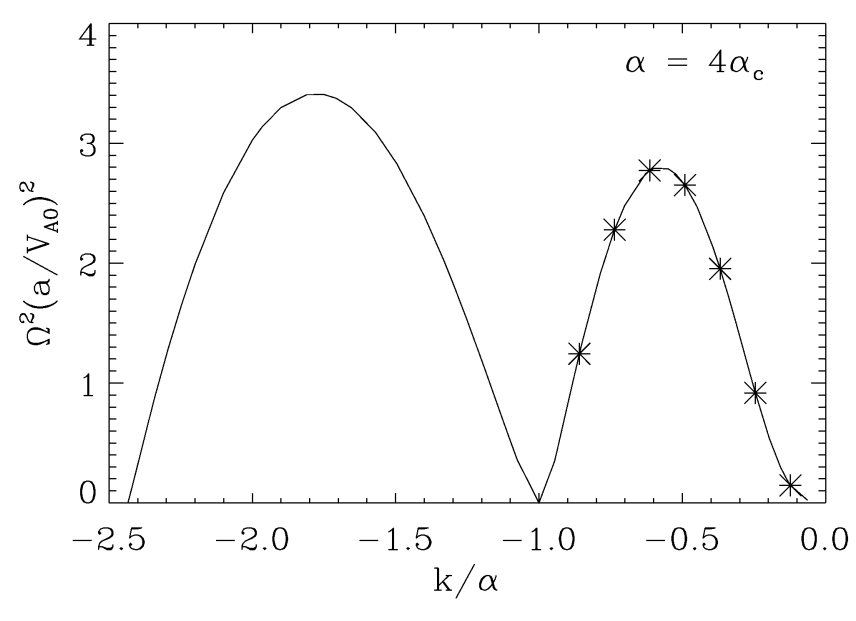

FIG. 1.-Linear growth rate $\Omega$ of unstable helical kink motion as a function of axial wavenumber $k$ for a twisted magnetic flux tube described by eqs. (1) and (2), with $\alpha=4 \alpha_{c}$. The asterisks represent the modes that are included in the initial perturbation of the buoyant flux tube in the simulation. The wavenumbers of these modes are $k_{j}=2 \pi j / L_{x}$, with $j=1,2,3,4,5,6,7$, and $L_{x}$ being the length of the flux tube in the simulation box.

kink mode is significantly less than the rise time of the tube. In a later paper, we will investigate the rise of flux tubes with initial twist that is near or slightly below the critical limit for the onset of the kink instability. It has been argued (Parker 1979; Linton et al. 1996; Emonet 1997) that as a horizontal flux tube rises and expands, the longitudinal field decreases at a faster rate ( $B_{x} \propto R^{-2}$, with $R$ being the tube radius) than the transverse field $\left(B_{\theta} \propto R^{-1}\right)$, leading to a decrease of critical $\alpha_{c}$ and therefore making the tube more kink unstable.

Since the dynamic timescale of interest is determined by the Alfvén speed, which is orders of magnitude smaller than the sound speed in the deep convection zone (the plasma $\beta$ is on the order of $10^{5}$ ), it is advantageous to use the anelastic formulation of the three-dimensional MHD equations, which corresponds to the limit of $\beta \rightarrow \infty$ (i.e., the sound speed is effectively infinite). The set of three-dimensional anelastic equations that we solve are given in Fan et al. (1998, eqs. [1]-[7]). For the numerical simulations, the three-dimensional magnetic field $\boldsymbol{B}$ and momentum $\rho_{0} \boldsymbol{v}$ are written in terms of vector potentials so as to guarantee that they are divergence free (see, e.g., Glatzmaier 1984): $\boldsymbol{B}=\boldsymbol{\nabla} \times \boldsymbol{\nabla} \times(\mathscr{B} \hat{z})+\boldsymbol{\nabla} \times(\mathcal{G} \hat{z}), \quad \rho_{0} \boldsymbol{v}=$ $\boldsymbol{\nabla} \times \boldsymbol{\nabla} \times(\mathcal{W} \hat{z})+\boldsymbol{\nabla} \times(\mathcal{Z} \hat{z})$, where $\hat{z}$ is the unit vector of the vertical direction. The background fluid is a plane-parallel, adiabatically stratified polytrope (so that the fluid is marginally stable to convection) with the total vertical height $d$ of the simulation domain spanning 3.45 density scale heights. The ratio of density between the top and bottom is 0.03 . The two horizontal dimensions of the Cartesian simulation domain are both $d / 2$ in length. The boundary conditions for the simulation box are periodic in the two horizontal directions and nonpenetrating no-stress for the velocities at the top and lower boundaries. We use a uniform grid of $128 \times 128 \times 256$ for the $d / 2 \times d / 2 \times d$ domain. The governing equations (eqs. [1]-[7] in Fan et al. 1998) explicitly include viscosity, magnetic diffusivity, and thermal diffusion. Like all numerical studies that are limited by the finite resolution of the simulation grid, the effective values of these diffusive parameters are generally much greater than those expected under solar conditions. Hence, the interpretation of the numerical results will be subject to the uncertainties of the effects of the unresolved small-scale dynamics. For our numerical simulations, the important nondimensional parameters are the viscous Lundquist number $S_{v} \equiv \rho_{r} v_{\mathrm{A}_{0}} a / \mu$ and the resistive Lundquist number $S_{r} \equiv$ $v_{\mathrm{A}_{0}} a / \eta$, where $\rho_{r}$ is the density at the bottom of the domain, $v_{\mathrm{A}_{0}}$ is the Alfvén speed at the axis of the initial flux tube, $a$ is the initial tube radius defined in equation (1), and $\mu$ and $\eta$ are respectively the dynamic viscosity and magnetic diffusivity, which are constant in the simulation domain. The values of $S_{v}$ and $S_{r}$ are respectively 43 and 4300 . The much larger value of $S_{r}$ is used to ensure that the flux "frozen-in" condition is approximately satisfied, while the low value of $S_{v}$ ensures that sharp velocity structures do not form. We set thermal diffusivity to a low value so that there is a negligible amount of heat diffusion across the flux tube during its evolution. We have done many tests to confirm the reliability of the code. One test is a comparison of the results of our code with that used in Linton et al. (1998a), which is a fully compressible MHD code using very different numerical algorithms. We found that in the absence of gravity and stratification, our code produces results that are in good agreement (better than 2\%) with that of Linton et al. (1998a) on the nonlinear evolution of a kinkunstable flux tube.

\section{SIMULATION RESULTS}

\subsection{Initial Conditions}

The initial flux tube is cylindrical with magnetic field profiles described by equation (1). Such a flux tube sitting in a gravitationally stratified layer has a mechanical equilibrium state in which the density difference (between the tube and the external medium) $\rho_{1}$ is equal to zero, so that the buoyancy force is zero and the gradient from the pressure perturbation $p_{1}$ balances the magnetic forces: $-\nabla p_{1}+(1 / 4 \pi)(\boldsymbol{B} \cdot \boldsymbol{\nabla}) \boldsymbol{B}-$ $\nabla\left(B^{2} / 8 \pi\right)=0$. The flux tube sits initially at a height of about one-fifth of the total height of the simulation domain, and the initial radius $a$ of the tube is 0.085 times the pressure scale height at the bottom.

To set the flux tube in motion, we make it buoyant by adding an entropy perturbation to the above mechanical equilibrium state of the tube:

$$
s^{\prime}=s_{0}^{\prime}(r)+\sum_{k_{j}} s_{k_{j}}^{\prime}(r) \cos \left(k_{j} x+\phi_{j}\right) .
$$

The entropy perturbation contains a component $s_{0}^{\prime}(r)$ that is constant along the tube axis ( $x$-direction) and a set of components that vary sinusoidally along the axis with wavenumbers $k_{j}=2 \pi j / L_{x}$, where $L_{x}$ is the length of the flux tube and $j=$ $1,2,3,4,5,6,7$. These wavenumbers $k_{j}$ correspond to the unstable kink modes represented by the asterisks in Figure 1. The profiles of $s_{0}^{\prime}(r)$ and $s_{k_{j}}^{\prime}(r)$ are Gaussians with the peak amplitudes of $s_{k_{j}}^{\prime}(r) \propto 1 / k_{j}$ and are all much smaller than the peak amplitude of $s_{0}^{\prime}(r)$. [The amplitude of the largest $s_{k_{j}}^{\prime}(r)$ is 0.025 of that of $s_{0}^{\prime}(r)$.] The phases $\phi_{j}$ of each $k_{j}$ mode are such that they all add constructively, i.e., $k_{j} x+\phi_{j}=0$, at the middle cross section of the tube. The middle of the tube is thus the most buoyant. As an average for the entire tube, the initial field-weighted mean buoyancy acceleration is $0.36 B_{x 0}^{2} /\left(4 \pi \rho_{r} H_{r}\right)$, where $\rho_{r}$ and $H_{r}$ are respectively the density and pressure scale height at the bottom of the domain. The variation of buoyancy along the tube axis will drive planar undulation of the flux tube. Each sinusoidal undulating mode can be decomposed into two helical kink modes of opposite 

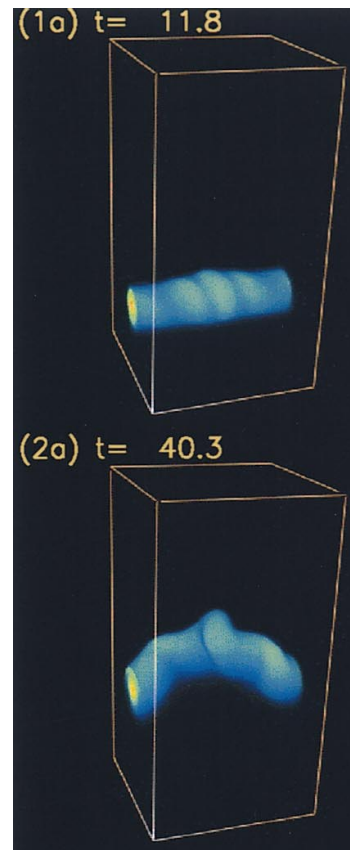

(3a) $t=67.2$

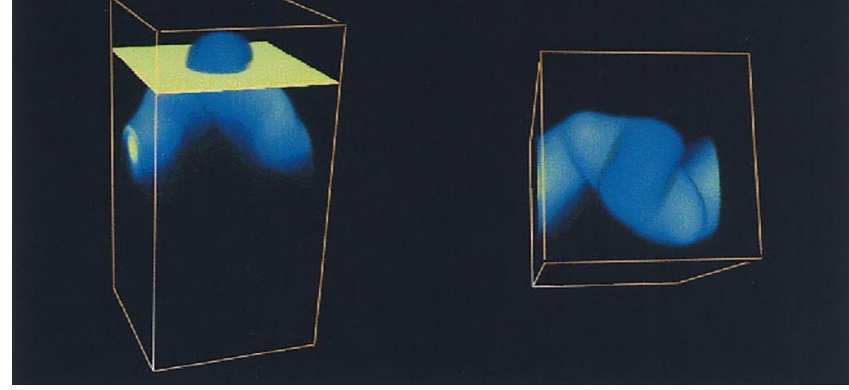

FIG. 2.-A series of snapshots of the flux tube during its rise as viewed from the side (left) and from the top (right). The color shading indicates the absolute magnetic field strength. The marked times are in units of $a / v_{\mathrm{A}_{0}}$. A cross section indicated by the green plane in panel $3 \mathrm{a}$ is shown in Fig. 4.

handedness. The one with the same handedness as that of the twisted field lines is unstable and will be excited.

\subsection{Evolution of the Tube}

Figure 2 shows several snapshots of the flux tube during its rise. The color in the images indicates the absolute magnetic field strength $B$. The marked times are in units of the Alfvén crossing timescale $a / v_{\mathrm{A}_{0}}$. Because of the high growth rates $(e-$ folding times $\sim a / v_{\mathrm{A}_{0}}$ ) of the unstable kink modes (see Fig. 1), the flux tube becomes kinked before it has risen any noticeable distance. The axis of the flux tube evolves into a helix as a result of the growth of multiple kink modes. The helix is of the same sense of handedness as the twisted field lines of the initial cylindrical tube. Figure 3 shows the evolution of the total magnetic and kinetic energies in the simulation domain, all normalized to the initial magnetic energy, and the evolution of the field-weighted average rising velocity $\left\langle v_{z}\right\rangle$, which reflects the mean rising velocity of the tube as a whole. The initial kink development of the flux tube (from $t=0$ to roughly $\left.t=13 a / v_{\mathrm{A}_{0}}\right)$ is similar to the results described in the simulations of Linton et al. (1998b), who study the kink instability of flux tubes in a uniform fluid without gravity and stratification. The qualitative behavior during this stage is that the unstable kink

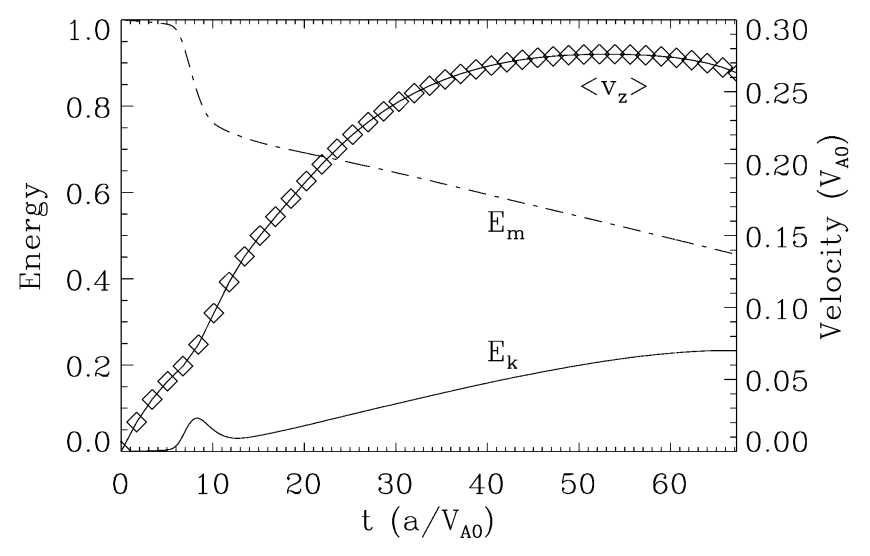

FIG. 3.-Evolution of total magnetic and kinetic energies, normalized to the initial magnetic energy, and the evolution of the field strength-weighted mean rising velocity $\left\langle v_{z}\right\rangle \equiv\left(\int B v_{z} d V\right) /\left(\int B d V\right)$ of the flux tube. Here $B$ is the absolute magnetic field strength, and $V$ denotes volume.

modes first grow exponentially, and after the linear phase that lasts several Alfvén crossing times, the kink saturates (as indicated by the first peak in the kinetic energy in Fig. 3). It can be seen in Figure 3 that the magnetic energy drops sharply as a result of the kink development. The decrease in magnetic energy is partly converted to the kinetic energy of the kink motion and partly goes to heating through direct Ohmic dissipation. After the kink saturates, the kinetic energy decreases. However, because of the presence of the driving buoyancy force, the flux tube does not settle into a stable kinked equilibrium as was found in Linton et al. (1998b). After a brief decline, the kinetic energy continues to rise steadily as a result of the buoyancy-driven acceleration of the flux tube.

After the initial stage of the rapid kink development, the dominant modes of motion in the subsequent evolution of the tube are its overall rise plus the kink mode of the lowest wavenumber $k_{1}=2 \pi / L_{x}$, which causes the tube to arch (see panels $2 \mathrm{a}$ and $3 \mathrm{a}$ of Fig. 2). Furthermore, we find that as the tube rises, the three major kinks on the tube continue to deform in such a way as to make the tube axis at these portions rotate farther away from the original $+x$-direction (see panels $2 \mathrm{~b}$ and $3 \mathrm{~b}$ of Fig. 2). This buckling deformation is most prominent at the apex of the tube. By $t=40 a / v_{\mathrm{A}_{0}}$, the tube axis at the apex has already been bent by more than $90^{\circ}$ away from the $+x$ direction. Hence, we expect that the emergence of this buckled upper portion of the flux tube should give rise to a magnetic region with polarity orientation inverted from Hale's polarity law. Figure 4 shows a horizontal cross section of the upper buckled portion of the flux tube at time $t=67.2$ (see panel 3a of Fig. 2, where the green plane indicates the height of the cross section). The contours in Figure 4 denote the vertical magnetic field and the arrows show the horizontal field. Solid line (dotted line) contours represent positive (negative) $B_{z}$. We see a very tight bipolar structure with the polarity orientation (i.e., the direction of the line drawn from the peak of the positive pole to the peak of the negative pole) rotated by about $145^{\circ}$ away from the $+x$-direction, which would be the normal polarity orientation expected from the direct emergence of the unkinked tube. The transverse field near the neutral line is sheared, although not as much as is often observed in $\delta$ spots where the transverse field runs nearly parallel to the neutral line. As of the last time in Figure 2, the flux tube is still entirely submerged beneath the surface in a pressure-dominated plasma 


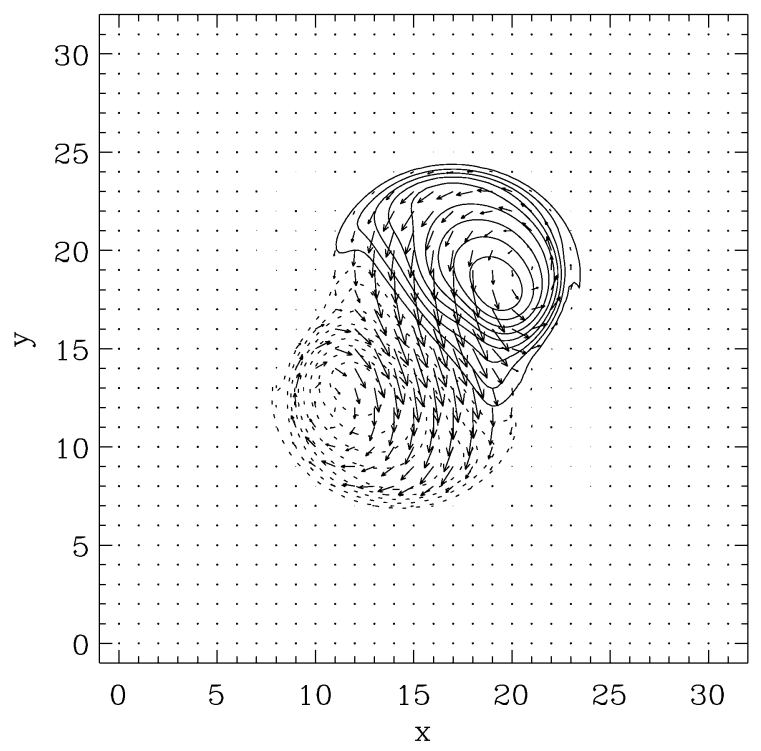

FIG. 4.-Horizontal cross section of the upper buckled portion of the flux tube at $t=67.2$ (see panel 3a of Fig. 2). The contours denote the vertical magnetic field $B_{z}$ with solid line (dotted line) contours representing positive (negative) $B_{z}$. The arrows show the horizontal magnetic field.

and is not yet significantly affected by the impenetrable upper boundary. The emergence of the flux tube through the surface into the low- $\beta$ plasma of the atmosphere is a complicated dynamical process that is beyond the scope of the current model. Extrapolating from the structure of the rising flux tube as it approaches the upper boundary, we speculate that the basic reversed polarity configuration of the vertical field will be seen at the photosphere as the tube is breaking through the surface, but the orientation of the transverse field at the photosphere will be more dependent upon how the field lines readjust themselves in the atmosphere.

Figure 5 shows the evolution of the magnetic field strength $B$ at the apex of the tube axis (see diamonds) as a function of height. In comparison, the dash-dotted line shows how the axial field strength $B$ of a thin, horizontal (unkinked) flux tube is expected to vary with height. For the rise and expansion of a thin, horizontal flux tube, the quantities $B R^{2}$ (axial flux, where $R$ is the tube radius) and $\rho R^{2}$ (mass per unit length) are conserved. Therefore, $B$ should vary as $1 / \rho$, which is the result represented by the dash-dotted line. It can be seen from Figure 5 that the kink development enhances the axial field strength by stretching the tube axis as it evolves into a helical shape.

The evolution of the averaged rise velocity $\left\langle v_{z}\right\rangle$ of the tube can be divided into an acceleration phase and a steady rise phase (see Fig. 3). The initial acceleration of $\left\langle v_{z}\right\rangle$ is consistent with the initial buoyancy divided by an added inertia of twice the fluid density (Lamb 1945). After the tube becomes kinked, the buoyancy of the tube is increased substantially (by about a factor of 2.5) due to heating and to an increase of the axial field strength. Eventually the rise of the flux tube reaches a steady speed as the drag force becomes comparable to the buoyancy. The present three-dimensional simulation is more viscous in comparison to previous two-dimensional simulations (Fan et al. 1998). The Reynolds number $R_{e}$ for the flux tube as estimated from the steady rise velocity, the radius of the tube, and the viscosity is only $\sim 10$, whereas $R_{e} \sim 100$ in Fan et al. (1998). By considering a balance of the mean buoyancy

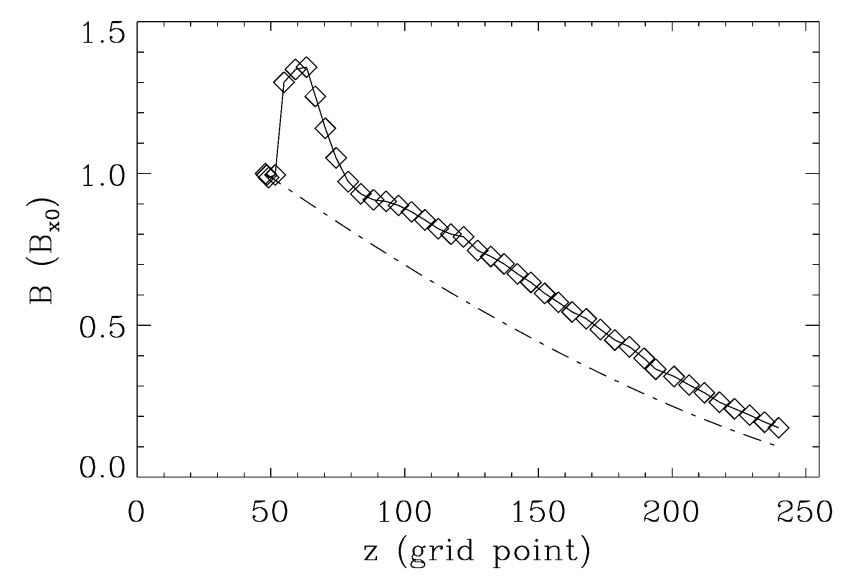

FIG. 5.-Diamonds show the evolution of field strength $B$ at the apex of the tube axis as a function of height. In comparison, the dash-dotted line corresponds to $B \propto 1 / \rho$, which describes how the axial field strength of a thin, horizontal (unkinked) flux tube is expected to vary with height.

and drag at the steady phase of $\left\langle v_{z}\right\rangle$, we find an effective drag coefficient $C_{D}$ of about 3 for the tube. It is a concern that the substantial heating (which increases the buoyancy of the tube) experienced by the flux tube may be an artifact due to the low Reynolds number in the simulation. However, in the limit of large Reynolds numbers where turbulence develops, it may be expected that the rate of dissipation of kinetic energy into heat becomes essentially independent of the Reynolds number, similar to the behavior of the drag coefficient $C_{D}$, which reaches a limiting value of $\sim 1$ (Batchelor 1967; Landau \& Lifshitz 1959). Therefore significant dissipation of the kinetic energy of the kink and rise motion may still take place. On the other hand, we note that the result of flux tube buckling does not depend on the effect of heating. To test this, we repeated the simulation, but this time omitting the viscous and Ohmic heating terms in the energy equation so that energy dissipation does not result in heating and an increase in buoyancy. Although the overall rise is slower, the flux tube still evolves into the buckled configuration.

\section{SUMMARY}

Through numerical simulation, we have studied the buoyant rise of a twisted, kink-unstable flux tube in an adiabatically stratified layer. We find that the kink development in conjunction with the buoyant rise leads to buckling of the top portion of the flux tube where the tube axis is being deflected more than $90^{\circ}$ from the original orientation. The emergence of such a buckled flux tube can lead to the formation of a compact magnetic bipole with polarity order reversed from Hale's polarity law. The need for toroidal flux tubes to have a minimum amount of twist (which is only $\sim 20 \%$ of $\alpha_{c}$ ) to sustain their cohesion as they traverse through the convection zone to form coherent active regions has been demonstrated in previous twodimensional simulations (Moreno-Insertis \& Emonet 1996; Fan et al. 1998). It may be the case that the subsurface origin of $\delta$ spot regions is a special class of flux tubes that through some means have acquired an unusually high amount of twist (with $\alpha$ near or greater than $\alpha_{c}$ ).

This work has been supported by NSF grant AST95-21779 and NASA Space Physics Theory Program. 


\section{REFERENCES}

Batchelor, G. K. 1967, An Introduction to Fluid Dynamics (Cambridge: Cambridge Univ. Press), 340

Emonet, T. 1997, Ph.D. thesis, Univ. La Laguna

Fan, Y., Zweibel, E. G., \& Lantz, S. R. 1998, ApJ, 493, 480

Freidberg, J. P. 1982, Rev. Mod. Phys., 54, 801

Glatzmaier, G. A. 1984, J. Comp. Phys., 55, 461

Lamb, H. 1945, Hydrodynamics (New York: Dover), 76

Landau, L. D., \& Lifshitz, E. M. 1959, Fluid Mechanics (London: Pergamon), 116

Leka, K. D., Canfield, R. C., McClymont, A. N., \& van Driel-Gesztelyi, L. 1996, ApJ, 462, 547

Linton, M. G., Dahlburg, R. B., Fan, Y., \& Fisher, G. H. 1998a, in preparation

Linton, M. G., Dahlburg, R. B., Fisher, G. H., \& Longcope, D. W. 1998b, $\mathrm{ApJ}$, in press
Linton, M. G., Longcope, D. W., \& Fisher, G. H. 1996, ApJ, 469, 954

Lites, B. W., Low, B. C., Martinez Pillet, V., Seagraves, P., Skumanich, A., Frank, Z. A., Shine, R. A., \& Tsuneta, S. 1995, ApJ, 446, 877

Low, B. C. 1996, Sol. Phys., 167, 217

Matsumoto, R., Tajima, T., Chou, W., Okubo, A., \& Shibata, K. 1998, ApJ, 493, L43

McClymont, A. N., \& Fisher, G. H. 1989, in Solar System Plasma Physics, ed. J. H. Waite, J. L. Burch, \& R. L. Moore (Geophysical Monograph 54; Washington DC: American Geophysical Union), 219

Moreno-Insertis, F., \& Emonet, T. 1996, ApJ, 472, L53

Parker, E. N. 1975, ApJ, 198, 205

. 1979, Cosmical Magnetic Fields (Oxford: Oxford Univ. Press), Chap. 9

Tanaka, K. 1991, Sol. Phys., 136, 133 